\title{
Evaluación electroquímica de soldaduras en tubos capilares de Incoloy 825
}

\section{Electrochemical evaluation of Incoloy 825 welds tubs}

\author{
Javier E. Rodríguez-Yáñez¹, Ericka Saborío-Leiva², \\ Daniel Mora-Montoya ${ }^{3}$
}

Fecha de recepción: 17 de abril del 2015

Fecha de aprobación: 21 de agosto del 2015

Rodríguez-Yáñez, J; Saborío-Leiva, E; Mora-Montoya, D. Evaluación electroquímica de soldaduras en tubos capilares de Incoloy 825. Tecnología en Marcha. Vol. 29, № 2, AbrilJunio 2016. Pág 85-94.

1 Ingeniero Químico. Investigador del Centro de Investigación en Corrosión, Instituto Costarricense de Electricidad. Apdo. 10032-1000, San José, Costa Rica; correo electrónico: urutico@gmail.com.

2 Ingeniera Metalúrgica. Investigadora del Centro de Investigación en Corrosión, Instituto Costarricense de Electricidad. Apdo. 10032-1000, San José, Costa Rica; correo electrónico: esaborio@ice.go.cr

3 Ingeniero en Materiales. Investigador de la Escuela de Ingeniería de Materiales, Instituto Tecnológico de Costa Rica. Apdo. 159-7050, Cartago, Costa Rica. 


\title{
Palabras clave
}

Incoloy 825; soldadura de orbital autógena; Tafel; geotermia; pozo ácido.

\section{Resumen}

La recuperación de los capilares de Incoloy 825 utilizados en pozos geotérmicos ácidos, por medio de soldadura orbital autógena, se evalúa en este estudio desde el punto de vista de su resistencia a la corrosión, en las condiciones de uso. El análisis se realiza por medio de técnicas electroquímicas básicas.

Se comparan los capilares soldados, con y sin tratamientos térmicos, respecto a capilares nuevos y usados.

La evaluación electroquímica indica la posibilidad de utilizar los capilares soldados de Incoloy 825 con tratamiento térmico para los pozos geotérmicos ácidos, desde el punto de vista de la resistencia a la corrosión.

\section{Keywords}

Incoloy 825; orbital welding; Tafel; geothermal; acid well.

\begin{abstract}
The resistance of corrosion of Incoloy 825 capillaries used in acid geothermal wells, by orbital welding, was evaluate in the conditions of use. The study was performe by means of basic electrochemical techniques. Also was analyzed new and old capillary soldiers, with or without thermal treatments

Electrochemical evaluation indicates the possibility of using capillary of Incoloy 825 welded, whit heat treated, for acidic geothermal wells, from the viewpoint of corrosion.
\end{abstract}

\section{Introducción}

El uso de los recursos geotérmicos para generar electricidad se ha incrementado en los últimos 20 años en Costa Rica, teniendo como principal desarrollador al Centro de Servicio de Recursos Geotérmicos (CSRG) del Instituto Costarricense de Electricidad (ICE). El proceso implica la extracción de aguas y vapor sobrecalentados, mediante pozos en acuíferos profundos calentados por la actividad magmática. La estabilidad del proceso y su continuidad es una de las características principales de la generación geotérmica. Dicha estabilidad depende de las políticas de explotación y del control de fenómenos secundarios. En los pozos geotérmicos ácidos se utilizan disoluciones de hidróxido de sodio $(\mathrm{NaOH})$ para neutralizar el fluido bifásico extraído, lo que aumenta el pH y disminuye la corrosión de los elementos del pozo (Mora Rodríguez, 2006).

Para aplicar dichos inhibidores se utilizan tubos capilares continuos de Incoloy 825, que resisten mejor las características fisicoquímicas de los fluidos geotérmicos con pH ácidos. La durabilidad de estos capilares depende de muchas variables pero en general se estima una vida útil de 6 a 10 meses. Los capilares que se pueden recuperar son los que presentan obstrucciones debido a la cristalización de la soda caústica, no aquellos que fallan por esfuerzo o por corrosión bajo tensión (stress corrosión cracking o SCC). Sobre estos se propusieron metodologías de soldado 
de dichos capilares y realizar ensayos comparativos respecto a un capilar nuevo y un capilar usado, mediante ensayos electroquímicos (Fontana, 1986; ASTM G 102-2010, 2010).

\section{Materiales y métodos}

\section{Capilares}

Los capilares de Incoloy 825 con un diámetro exterior de 9,54 mm y 1,58 mm de espesor que se utilizaron en los pozos ácidos del Campo Geotérmico Miravalles presentan la composición química descrita en la cuadro 1, según la información proporcionada por los proveedores del CSRG (Mora Rodríguez, 2006).

Previamente a la soldadura de los capilares se realizó una limpieza con acetona grado reactivo (95\%) para la remoción de grasas o hidrocarburos, luego se lijaron los extremos a soldar con una lija No. 400 Mesh. Para dicha aleación no fue necesario un decapado, ya que la formación de óxidos superficiales es mínima (ASTM A 380-06, 2006).

Cuadro 1. Composición química porcentual de los capilares de Incoloy 825

\begin{tabular}{|c|c|c|c|c|c|c|c|}
\hline \multicolumn{7}{|c|}{ Composición química porcentual (\%) } \\
\hline $\mathrm{C}$ & $\mathrm{Si}$ & $\mathrm{Mn}$ & $\mathrm{Cr}$ & $\mathrm{Ni}$ & $\mathrm{Mo}$ & $\mathrm{Cu}$ & $\mathrm{Ti}$ \\
\hline 0,03 & 0,5 & 0,8 & 21,5 & 40 & 3 & 1,7 & 0,8 \\
\hline
\end{tabular}

Después de la limpieza y soldadura, las muestras se sometieron a tratamientos térmicos de alivio de tensiones a $410^{\circ} \mathrm{C}$ y $955^{\circ} \mathrm{C}$, según lo descrito por el International Metal Handbook de la ASM (ASM, 1990). Los tratamientos térmicos (TT) se realizaron en un horno Lindberg Blue M $1200{ }^{\circ} \mathrm{C}$, modelo número HTF55342C, durante 17 minutos. Se obtuvieron de esta manera dos capilares soldados a distintas temperaturas que se identificaron como Soldado TT 410 y Soldado TT 955.

Los resultados se compararon con las muestras sin TT, considerando capilares nuevos (Nuevo), capilares usados en pozo (Usado) y capilares soldados (Soldado $\sin T T$ ).

\section{Disoluciones}

Para el proceso de evaluación electroquímica, como electrolito para los capilares se utilizaron las disoluciones a las que están expuestos habitualmente en los pozos geotérmicos ácidos: solución de $\mathrm{NaOH}$ al $40 \%$ a partir de una disolución comercial de $\mathrm{NaOH}$ al $50 \%$ (m/v) y la salmuera ácida del pozo 02.

La salmuera del pozo 02 presenta una composición química ácida, con valores altos de cloruros y sulfatos. La composición de su fase líquida puede verse en la cuadro 2 (Mora Rodríguez, 2006).

Para el pulido electroquímico, previo a la evaluación metalográfica, se utilizó como reactivo de ataque ácido nítrico concentrado (60\% y densidad de $\left.1,37 \mathrm{~g} / \mathrm{cm}^{3}\right)$, marca J. T. Backer, mezclado con ácido acético glacial (99\%) marca Panreac y agua destilada en proporción 2/1/17 (v/v), respectivamente. Para la disolución electrolítica se generó una mezcla de metanol absoluto (95\%), marca J. T. Backer, y ácido perclórico (70\% y densidad $1.67 \mathrm{~g} / \mathrm{cm}^{3}$ ), marca Merck, en proporción 2/1 (v/v) (ASTM A 380-06, 2006; ASTM 407-07, 2007) 
Todas las disoluciones fueron elaboradas con aguas de calidad tipo IV según la norma ASTM 1193-2001 (con conductividad menor a $5 \mu \mathrm{S} / \mathrm{cm}$, pH entre 5 y 8, así como concentraciones de $\mathrm{Na}$ y Cl menores a 50 mg/l) (ASTM D1193-06 (2011), 2011).

Cuadro 2. Composición de la salmuera ácida del pozo PGM Nº2, medidas de la fase líquida a $98{ }^{\circ} \mathrm{C}$

\begin{tabular}{|c|c|}
\hline Pozo & PGM-02 \\
\hline Fecha & 29-nov-2011 \\
\hline $\mathrm{pH}$ & 3,04 \\
\hline Cond. $(\mu S / \mathrm{cm})$ & 12720 \\
\hline $\mathrm{Na}(\mathrm{ppm})$ & 2471 \\
\hline K (ppm) & 333 \\
\hline $\mathrm{Ca}(\mathrm{ppm})$ & 39 \\
\hline Mg (ppm) & 7,05 \\
\hline Fe total (ppm) & 23,2 \\
\hline $\mathrm{Cl}(\mathrm{ppm})$ & 4042 \\
\hline $\mathrm{SO}_{4}^{-2}(\mathrm{ppm})$ & 315 \\
\hline $\mathrm{HCO}_{3}^{-}(\mathrm{ppm})$ & nd \\
\hline$F(p p m)$ & 2,59 \\
\hline B (ppm) & 69 \\
\hline $\mathrm{H}_{2} \mathrm{~S}(\mathrm{ppm})$ & 2,04 \\
\hline $\mathrm{NH}_{3}(\mathrm{ppm})$ & 13,5 \\
\hline As (ppm) & 10,5 \\
\hline $\mathrm{SiO}_{2}$ monomérica (ppm) & 538 \\
\hline $\mathrm{SiO}_{2}$ total $(\mathrm{ppm})$ & 616 \\
\hline S.T.D (ppm) & 8050 \\
\hline
\end{tabular}

Nota: nd, no detectable.

\section{Ensayos electroquímicos}

La metodología utilizada para evaluar la corrosión es la siguiente (Fontana, 1986; González, 1989):

- Potencial de circuito abierto $\left(\mathrm{E}_{\mathrm{oc}}\right)$, durante 10 minutos.

- Resistencia de polarización lineal $\left(R_{p}\right)$, mediante la aplicación de un barrido de potencial a $10 \mathrm{mV} / \mathrm{min}$ entre $-20 \mathrm{mV}$ y $+20 \mathrm{mV}$ respecto a $\mathrm{E}_{\text {oc }}$.

- Curvas de Tafel catódica y anódica, con un barrido de potencial de $50 \mathrm{mV} / \mathrm{min}$, entre los $-250 \mathrm{mV}$ y los $+250 \mathrm{mV}$, respecto a $\mathrm{E}_{\text {oc }}$.

Las evaluaciones se realizaron a temperatura ambiente $\left(20^{\circ} \mathrm{C}\right)$, empleando un electrodo de referencia de $\mathrm{AgCl} / \mathrm{Ag}$ y un contraelectrodo de platino (Pt). Todos los potenciales se indican con base en el electrodo de referencia. 
Para hacer las mediciones electroquímicas se utilizó un potenciostato-galvanostato marca Gamry, modelo Reference 600.

Los valores de Rp se estiman gráficamente de la pendiente media en el rango de análisis.

Las pendientes de Tafel se calculan gráficamente en el rango lineal entre los (60 a 200) mV, respecto $a \mathrm{E}_{\mathrm{oc}}$, en cada rama de dichas curvas, considerando los valores más cercanos a valores cada $30 \mathrm{mV} / \mathrm{dec}$, asociados a cada condición cinética de intercambio de electrones.

Las velocidades de corrosión $\left(\mathrm{V}_{\text {corr }}\right)$ se calculan a partir de los valores de $\mathrm{Rp}$, las áreas geométricas expuestas y las pendientes de Tafel, según la ecuación general de Stern-Geary (ASTM G 102-2010, 2010; ASTM G5-2004, 2010; Bard \& Faulkner, 2001).

\section{Ecuación de Stern-Geary}

$$
j_{\text {corr }}=\frac{B}{A \times R_{p}}
$$

donde:

Rp: es la resistencia a la polarización, en W

$\mathrm{j}_{\text {corr }}$ : es la densidad de corriente de corrosión en $\mathrm{A} / \mathrm{cm}^{2}$ (o C/s cm²).

B: es la constante de Stern-Geary, que para cálculos simples se estima en 26 mV, que también puede calcularse como:

$$
B=b_{a} \times b_{c} /\left(2,3 \times\left(b_{a}+b_{c}\right)\right)
$$

donde:

$b_{a}$ : es la pendiente de Tafel de la zona anódica.

$b_{c}$ : es la pendiente de Tafel de la zona catódica.

$$
V_{\text {Corr }}=\frac{K \times j_{\text {corr }} \times P M}{n \times F \times d}
$$

donde:

A: es el área expuesta en $\mathrm{cm}^{2}$

$V_{\text {corr }}$ : es la velocidad de corrosión, expresada como pérdida de espesor en mm/año.

$\mathrm{K}$ : es la constante de transformación de unidades, $10 \mathrm{~mm} / \mathrm{cm} \times 31536000$ s/año.

PM: es el peso molecular del metal en $\mathrm{g} / \mathrm{mol}, 56 \mathrm{~g} / \mathrm{mol}$ en Incoloy 825.

$\mathrm{d}$ : es la densidad del metal en $\mathrm{g} / \mathrm{cm}^{3}, 8,14 \mathrm{~g} / \mathrm{cm} 3$ en Incoloy 825 .

\section{Metalografías}

Se hizo la evaluación metalográfica de los capilares de Incoloy 825, inspeccionando la soldadura, la zona afectada térmicamente y el material base. Las probetas fueron pulidas y atacadas electrolíticamente, usando como electrolito de pulido una disolución de metanol absoluto y ácido nítrico, indicada anteriormente, durante 15 segundos a $55 \mathrm{~V}$. Como reactivo de ataque se utilizó una disolución de ácido acético, ácido nítrico y agua desionizada durante 40 segundos a $3 \mathrm{~V}$. La evaluación se realizó utilizando un microscopio metalográfico marca Olympus, modelo GX41, y el software analizador de imágenes Paxit IA44, marca Leco (ASM, 1990). 


\section{Resultados y discusión}

\section{Ensayos electroquímicos}

Se realizaron ensayos de corrosión de los capilares a las diferentes muestras indicadas, utilizando tres capilares diferentes para cada muestra tipo, a fin de determinar la variabilidad.

Los valores obtenidos en los ensayos electroquímicos con salmuera del pozo geotérmico ácido (pozo 02) se aprecian en la cuadro 3, mientras que para la disolución de $\mathrm{NaOH}$ al $40 \%$ se presentan en la cuadro 4. Estos son valores promedio de los distintos capilares evaluados, sin que se presentara una variabilidad significativa.

En general, desde el punto de vista termodinámico, se espera que el valor de $\mathrm{E}_{\mathrm{oc}}$ no se desplace a potenciales más positivos; mientras que respecto a la cinética, se espera que los

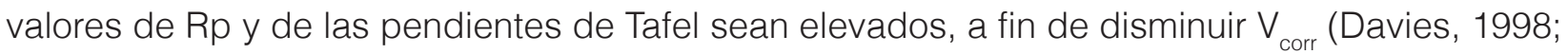
Schweitzer, 1986).

\section{En salmuera ácida}

En la cuadro 3 se aprecia que los valores de $E_{\text {oc }}$ de los capilares soldados (con y sin TT) se encuentran alrededor del valor del $\mathrm{E}_{\mathrm{oc}}$ de un capilar nuevo.

En el caso de los capilares soldados y con TT, los potenciales de $\mathrm{E}_{\mathrm{oc}}$ disminuyen con el aumento de la temperatura del tratamiento térmico y son más negativos que los capilares soldados y sin TT. Esto se relaciona con una mayor estabilidad termodinámica de los primeros.

Para el caso específico del capilar soldado con TT a $955^{\circ} \mathrm{C}$, los valores de potencial de $\mathrm{E}_{\text {oc }}$ son incluso inferiores a los potenciales de un capilar nuevo. El $\mathrm{E}_{\mathrm{oc}}$ del capilar usado presenta un valor alto, probablemente asociado a la exposición en el pozo.

Para el caso de los valores de Rp en los sistemas de capilares soldados, con y sin TT, se obtienen valores similares para los tres tipos de capilares, aunque crecientes al aumentar la temperatura del tratamiento térmico. Además, estos son menores en comparación con los capilares nuevos o usados.

Cuadro 3. Ensayos con salmuera del pozo 02 sobre capilares de Incoloy 825 de 9,52 mm de diámetro, nuevos, usados, soldados con y sin tratamiento térmico

\begin{tabular}{|c|c|c|c|c|c|c|c|}
\hline Muestra & $\begin{array}{c}\mathrm{E}_{\text {oc }} \\
(\mathrm{mV} \text { vs Ref })\end{array}$ & $\begin{array}{c}\mathrm{R}_{\mathrm{p}} \\
(\mathrm{Ohm})\end{array}$ & $\begin{array}{c}\beta_{\text {cat. }}(\mathrm{mV}) \\
\text { Nuevo }\end{array} \mathbf{\beta}_{\text {anód. }}(\mathrm{mV})$ & $\begin{array}{c}\mathrm{A} \\
\left(\mathrm{cm}^{2}\right)\end{array}$ & $\mathrm{j}_{\text {corr }}\left(\mu \mathrm{A} / \mathrm{cm}^{2}\right)$ & $\begin{array}{c}V_{\text {corr }}(\mathrm{mm} / \\
\text { año })\end{array}$ \\
\hline Usado & 145,2 & 11.640 & 210 & 210 & 48,04 & 0,082 & 0,69 \\
\hline Soldada sin TT & $-69,5$ & 3.545 & 210 & 180 & 37,77 & 0,315 & 3,54 \\
\hline Soldado TT 410 & $-88,3$ & 4.920 & 240 & 120 & 35,28 & 0,200 & 2,25 \\
\hline Soldado TT 955 & $-135,0$ & 6.138 & 210 & 180 & 35,28 & 0,195 & 2,19 \\
\hline
\end{tabular}




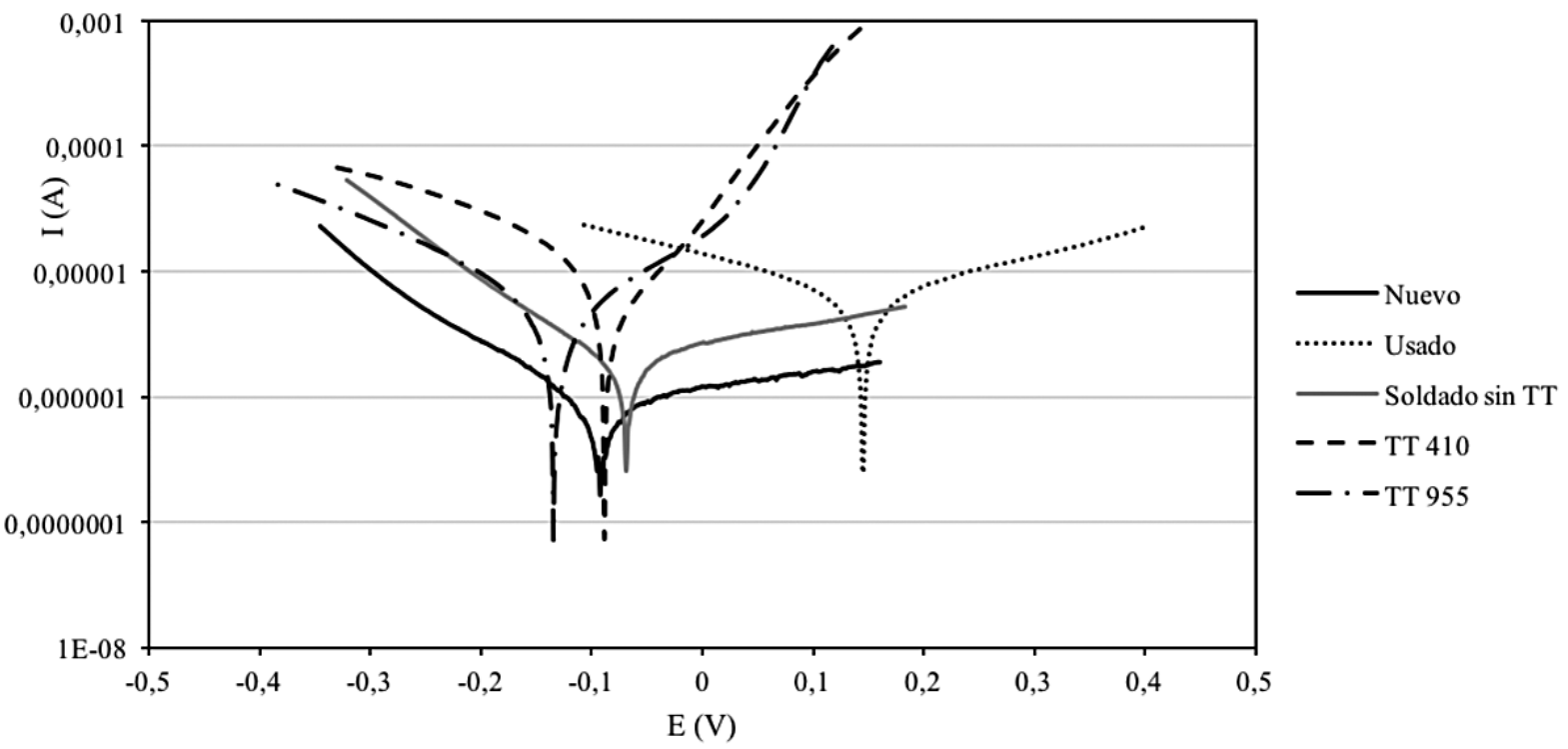

Figura 1. Curvas de Tafel para las distintas muestras evaluadas en salmuera del pozo 02, para Incoloy 825 de 9,52 mm de diámetro, línea continua capilar Nuevo, línea de rayas y puntos capilar Usado, línea gris capilar Soldado sin $T T$, línea de rayas capilar Soldado con $T T$ a $410^{\circ} \mathrm{C}$ y línea de puntos capilar Soldado con TT a $955^{\circ} \mathrm{C}$.

En las curvas de Tafel del cuadro 1 y la figura 1 se puede apreciar que las pendientes anódicas son menores para las curvas de los capilares soldados con y sin TT, especialmente hay una reducción importante para el TT a $410{ }^{\circ} \mathrm{C}$, mientras que el valor de la pendiente catódica aumenta ligeramente solo en el capilar con TT a $410^{\circ} \mathrm{C}$. Estas variaciones son indicativas de que la soldadura afecta la reacción anódica, haciéndola más favorable sobre la superficie de la soldadura.

A partir de los datos anteriormente analizados se observa que los capilares usados presentan una $V_{\text {corr }}$ similar al de un capilar nuevo, mientras que los capilares soldados (con y sin TT) presentan $V_{\text {corr }}$ superiores a estos.

Los capilares soldados disminuyen ligeramente su $\mathrm{V}_{\text {corr }}$ con los tratamientos térmicos.

Frente a una disolución de salmuera ácida, los capilares de Incoloy 825 soldados con TT muestran una leve mejora general de la $V_{\text {corr }}$ frente a capilar soldado sin TT, mejorando su $E_{o c}$ y $R p$, con poca variación, mientras que los valores de pendientes de Tafel son similares, afectando poco el valor de $\mathrm{V}_{\text {corr }}$. Entre ellos el que presenta mejores resultados es el TT a $955^{\circ} \mathrm{C}$.

\section{En solución de $\mathrm{NaOH}$}

Los ensayos electroquímicos desarrollados con la solución de $\mathrm{NaOH}$ al $40 \%$ (ver cuadro 4) muestran valores de $\mathrm{E}_{\mathrm{oc}}$ similares para los capilares nuevos y los capilares soldados sin TT; mientras que el capilar usado presenta un $\mathrm{E}_{\mathrm{oc}}$ menor, igual que el capilar con TT a $410{ }^{\circ} \mathrm{C}$. Por su parte, el capilar con TT a $955^{\circ} \mathrm{C}$ tiene un valor de $\mathrm{E}_{\mathrm{oc}}$ mayor que el capilar nuevo, lo cual se asocia con una mayor estabilidad termodinámica de la soldadura. 
Cuadro 4. Ensayos electroquímicos con $\mathrm{NaOH}$ al 40\%, sobre capilares de Incoloy 825 de 9,52 mm de diámetro, nuevos, usados y soldados, con y $\sin \mathrm{TT}$

\begin{tabular}{|c|c|c|c|c|c|c|c|}
\hline Muestra & $\begin{array}{c}\mathrm{E}_{\mathrm{oc}} \\
(\mathrm{mV} \text { vs Ref })\end{array}$ & $\begin{array}{c}\mathrm{Rp} \\
(\mathrm{Ohm})\end{array}$ & $\begin{array}{c}\beta_{\text {cat }}(\mathrm{mV}) \\
\text { Nuevo }\end{array}$ & $\begin{array}{c}\beta_{\text {anod }} \\
(\mathrm{mV})\end{array}$ & $\begin{array}{c}\mathrm{A} \\
\left(\mathrm{cm}^{2}\right)\end{array}$ & $\mathrm{j}_{\text {corr }}\left(\mu \mathrm{A} / \mathrm{cm}^{2}\right)$ & $\begin{array}{c}\mathrm{V}_{\text {corr }}(\mathrm{mm} / \\
\text { año })\end{array}$ \\
\hline Usado & $-642,4$ & 6.291 & 240 & 120 & 55,72 & 0,099 & 1,12 \\
\hline Soldada sin TT & $-684,0$ & 7.983 & 270 & 180 & 50,23 & 0,117 & 1,32 \\
\hline Soldado TT 410 & $-275,3$ & 10.180 & 270 & 240 & 37,77 & 0,144 & 1,61 \\
\hline Soldado TT 955 & $-1000,7$ & 11.750 & 240 & 120 & 35,28 & 0,084 & 0,94 \\
\hline
\end{tabular}

Los valores de Rp son similares para el capilar nuevo y el usado, pero resultan menores para el capilar soldado sin TT. Los capilares con TT aumentan el valor de Rp, siendo superiores que en un capilar nuevo y similares entre sí, aunque algo mayor para el TT a $955^{\circ} \mathrm{C}$.

Las pendientes catódicas de Tafel estimadas de la Figura 2 no varían mucho en los distintos capilares. En cambio, las pendientes anódicas son diferentes para cada situación, siendo elevadas en los capilares usados, los capilares soldados sin TT y con TT a $410{ }^{\circ} \mathrm{C}$, mientras que son bajas para capilares nuevos y soldados con TT a $955^{\circ} \mathrm{C}$. Esto se puede relacionar con un aumento de la cinética de la reacción anódica sobre la superficie, al aumentar la temperatura del tratamiento térmico en la soldadura.

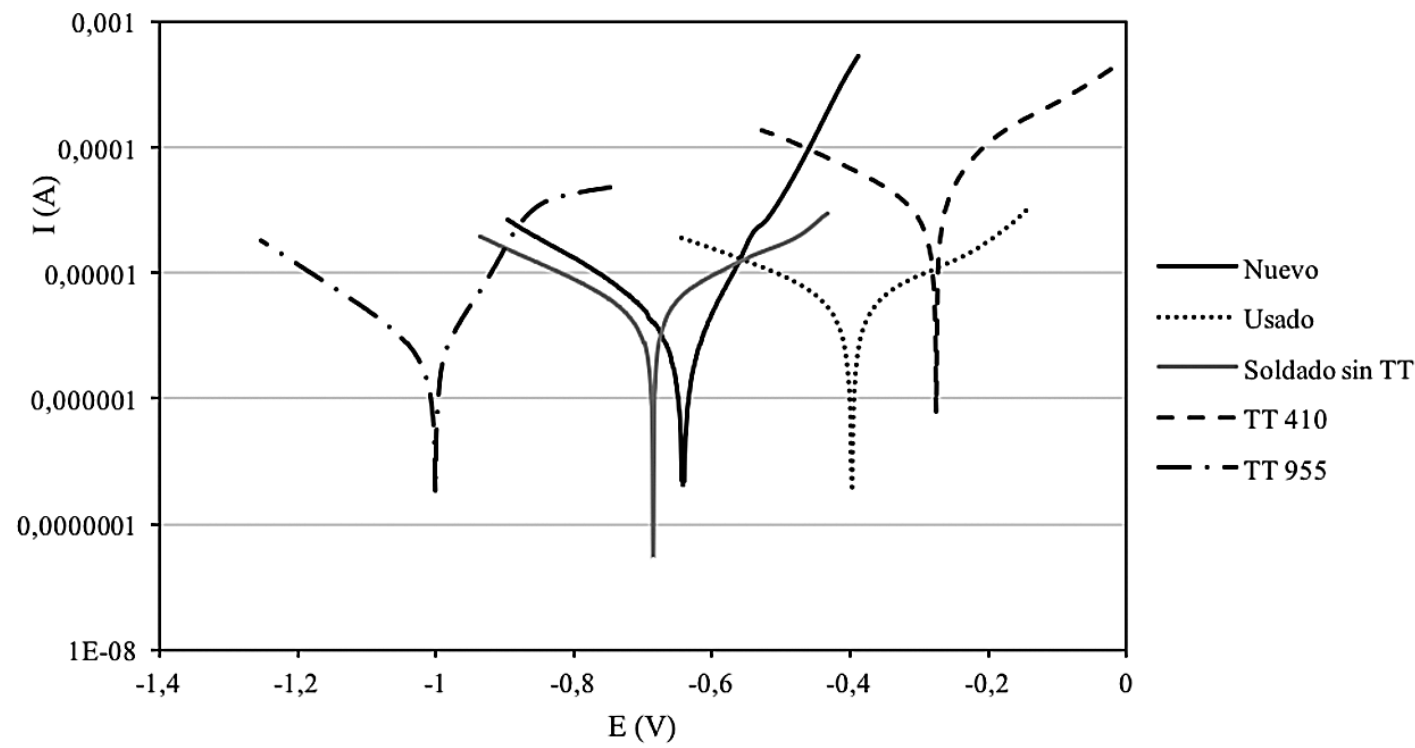

Figura 2. Curvas de Tafel para las distintas muestras evaluadas en $\mathrm{NaOH} 40 \%$, para Incoloy $825 \mathrm{de} 9,52 \mathrm{~mm}$ de diámetro, línea continua capilar Nuevo, línea de rayas y puntos capilar Usado, línea gris capilar Soldado sin TT, línea de rayas capilar Soldado con $T T$ a $410^{\circ} \mathrm{C}$ y línea de puntos capilar Soldado con TT a $955^{\circ} \mathrm{C}$. 
Los valores de $V_{\text {corr }}$ indican que el capilar nuevo y el capilar usado tienen una muy baja velocidad de corrosión frente a la disolución de $\mathrm{NaOH}$. Cuando se trata de capilares soldados, la $V_{\text {corr }}$ aumenta notablemente, pero se aprecia una disminución de la velocidad de corrosión en los capilares al aplicar los tratamientos térmicos.

De tal manera que el tratamiento térmico a $410^{\circ} \mathrm{C}$ aumenta el $\mathrm{E}_{\mathrm{oc}} \mathrm{y}$ el $\mathrm{R}_{\mathrm{p}}$, con pendientes de Tafel elevadas, lo que redunda en un $V_{\text {corr }}$ menor al del capilar soldado sin TT. Mientras que el TT a $955^{\circ} \mathrm{C}$ disminuye el $\mathrm{E}_{\mathrm{oc}}$ y a la vez aumenta el $\mathrm{Rp}$, lo que hace que la $\mathrm{V}_{\text {corr }}$ sea la menor de todos los sistemas analizados, aun frente al efecto de disminuir el valor de la pendiente de Tafel anódica.

\section{Análisis metalográfico}

Mediante análisis metalográfico se observa en la Figura 3 el capilar de Incoloy 825 previo al pulido electrolítico y al ataque, mostrando muy pocas inclusiones no metálicas. Posteriormente al ataque, se aprecia en la Figura 4 que la soldadura no produjo ningún precipitado de carburos de cromo en los límites de grano y que la estructura es de matriz austenítica, tanto para las soldaduras con tratamiento térmico como aquellas sin éste.

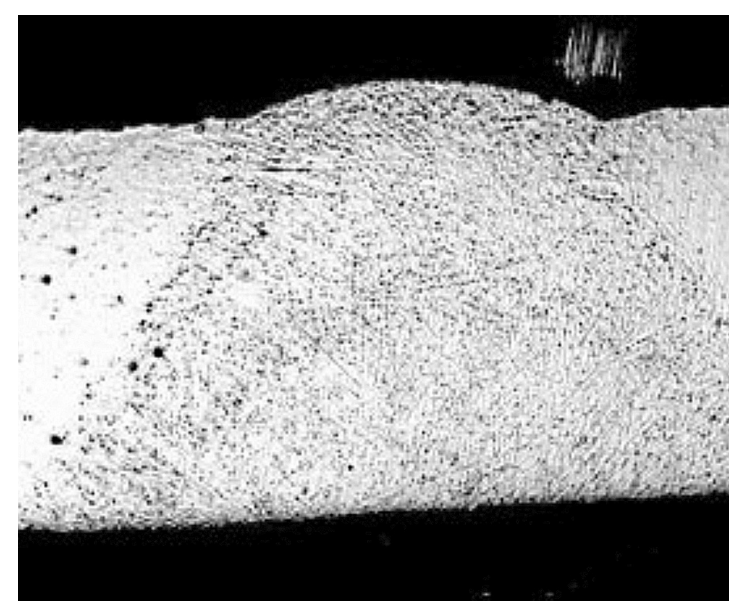

Figura 3. Soldadura en tubo capilar sin atacar, 50x.

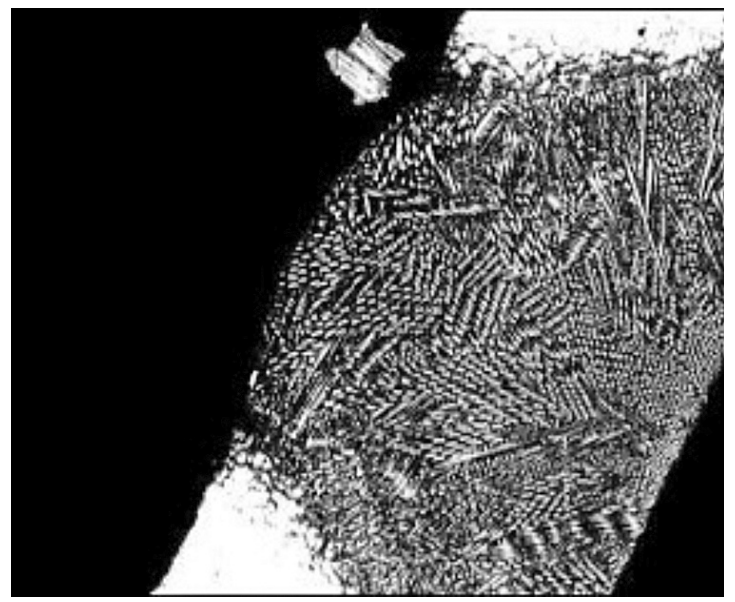

Figura 4. Soldadura en tubo capilar atacada con ácido acético, ácido nítrico y agua desionizada, 50x. 


\section{Conclusiones}

Los ensayos electroquímicos en los dos medios de tratamiento (salmuera ácida del pozo 02 y $\mathrm{NaOH}$ ) presentan valores de velocidad de corrosión del mismo orden de magnitud en capilares nuevos y usados. Paralelamente, se evidencia un efecto importante de los capilares soldados, donde el capilar soldado sin TT presenta valores de $\mathrm{V}_{\text {corr }}$ mayores que un capilar usado, pero la aplicación de los tratamientos térmicos disminuye la velocidad de corrosión, al aumentar la temperatura del tratamiento.

Estas variaciones de $V_{\text {corr }}$ en la salmuera ácida (pozo 02) se asocian a una disminución del valor de $\mathrm{E}_{\mathrm{oc}}$, con oscilaciones en los valores de las pendientes de Tafel; mientras que en la disolución de $\mathrm{NaOH}$ se asocian a disminuciones de las pendientes de Tafel, en especial en la rama anódica con $\mathrm{TT}$ a $955^{\circ} \mathrm{C}$, pero donde el comportamiento de $\mathrm{E}_{\mathrm{oc}}$ es variable.

La evaluación metalográfica indica que no se produce deposición de cromo o carburos en la región de la soldadura y áreas cercanas.

A través de esta investigación se plantea la factibilidad de usar capilares soldados con TT a $955^{\circ} \mathrm{C}$, en pozos geotérmicos ácidos para la adición de inhibidores, con la salvedad de que podrían tener una vida útil algo menor que un capilar nuevo, debido a la mayor $\mathrm{V}_{\text {corr }}$ de estos en las salmueras ácidas.

Debe analizarse la aplicación del TT respecto a su efecto sobre la reducción de tensiones debido a la soldadura, ya que el presente estudio solo se enfocó en la resistencia a la corrosión.

\section{Agradecimientos}

Se agradece de forma especial el apoyo brindado por el Centro de Servicios y Recursos Geotérmicos del ICE para el desarrollo de este proyecto.

\section{Bibliografía}

ASM. (1990). International Metal Handbook, Vol. 4. Heat Treating. Cleveland, American Society for Metals.

ASTM A 380-06. (2006). Standard Practice for Clearing, Descaling, and Passivation of Stainless Steel Parts. American Society for Testing and Materials.

ASTM 407-07. (2007). Standard Practice for Microetching. American Society for Testing and Materials.

ASTM G 102-2010. (2010). Standard Practice form Calculation of Corrosion Rates adn Related Information from Electrochemical Measurements. American Society for Testing and Materials. OJO: tienen la misma fecha, deben diferenciarse con a y $b$ e incluir $a$ y b en el texto.

ASTM G5-2004. (2010). Standard Reference Test Method form Making Potentiostatic and Potentiodynamic Anodic Polarization Measurements. American Society for Testing and Materials.

ASTM D1193-06 (2011). (2011). Standard Specification for Reagent Water. American Society for Testing and Materials.

Bard, A. \& Faulkner, L. (2001). Electrochemical Methods. New York: J. Wiley \& Sons Cia.

Davies, J. (1998). Metals Handbook. Ohio, EE.UU.: ASM International.

Fontana, M. (1986). Corrosion Engineering. EE.UU.: 3 ed. Custom Publishing, Mac Graw Hill.

González, J. (1989). Control de la Corrosión: Estudio y Medida por Técnicas Electroquímicas. Madrid: Centro Nacional de Investigaciones Metalúrgicas.

Mora Rodríguez, J. (2006). Programa de control del tubo capilar usado en los pozos del Campo Geotérmico Miravalles. Cartago, Costa Rica. Tesis de Maestría en Administración de la Ingeniería Electromecánica. Instituto Tecnológico de Costa Rica.

Schweitzer, P.A. (1986). Corrosion Resistance Tables. EE.UU.: Marcel Dekker. 